\title{
Evolution of Galaxies in Triaxial Halos with Figure Rotation
}

\author{
Kenji Bekki \\ School of physics, University of New South Wales, Sydney, NSW, 2052, \\ Australia \\ K. C. Freeman \\ Mount Stromlo \& Siding Spring Observatories, Australian National \\ University, Weston Creek P.O., ACT 2611, Australia
}

\begin{abstract}
Firstly, we demonstrate that unusually large outer HI spiral arms observed in NGC 2915 can form in an extended gas disk embedded in a massive triaxial dark matter halo with slow figure rotation, through the strong gravitational torque of the rotating halo. Secondly, we show that the figure rotation of a triaxial dark matter halo can influence dynamical evolution of disk galaxies by using fully self-consistent numerical simulations. We particularly describe the formation processes of "halotriggered" bars in thin galactic disks dominated by dark matter halos with figure rotation and discuss the origin of stellar bars in low luminosity, low surface brightness (LSB) disk galaxies. Thirdly, we provide several implications of the present numerical results in terms of triggering mechanism of starbursts in galaxies and stellar bar formation in high redshifts.
\end{abstract}

\section{Kinematics of dark matter halos and galaxy evolution}

Although several attempts have been so far made to reveal the shapes (e.g., the degree of oblateness or triaxiality) of dark matter halos in galaxies (e.g., Sackett \& Sparke 1990; Franx, van Gorkom, \& de Zeeuw 1994; Olling 1995; Salucci \& Persic 1997; Sackett 2003 in this conference), rotational properties of dark matter halos have been less discussed. Based on the detailed analysis of structure and kinematics of the very extended HI disk around NGC 2915, Bureau et al. (1999) first suggested that the observed spiral-like structures in the HI disk can be formed by a triaxial halo with figure rotation. However, it is unclear whether a triaxial dark halo with figure rotation is really responsible for the observed extended spiral structures in NGC 2915, because of the lack of numerical studies of gas dynamics in the gravitational potentials of triaxial halos with figure rotation. Also it is an important problem to understand how figure rotation of a dark matter halo in a disk galaxy influences dynamical evolution, star formation history, and chemical evolution of the disk, because previous theoretical/numerical studies so far have not extensively investigated such influence of figure rotation of dark matter halos. Therefore, we here investigate numerically (1) whether the observed NGC 2915's giant HI spirals can be formed by tidal force of the figure 
rotation of the triaxial dark halo and (2) how the figure rotation of a triaxial dark halo in a disk galaxy influences the formation of stellar bars and spiral arms within it.

\section{Evidence of a triaxial halo with figure rotation?}

We consider an extended uniform gas disk of a dwarf galaxy embedded in a triaxial dark matter halo with figure rotation. We adopt TREESPH codes described in Bekki (1997) for hydrodynamical evolution of galaxies and thereby investigate the dynamical evolution of the gas disk under the triaxial dark halo (The details of the model are given in Bekki \& Freeman 2002). The unusually extended HI gas in NGC 2915 is modeled as a uniform thin gas disk with the size (represented by $R_{\mathrm{g}}$ ) of $15 \mathrm{kpc}$ and the mass $\left(M_{\mathrm{g}}\right)$ of $10^{8} M_{\odot}$. The gas disk with uniform radial density distribution is represented by $20 \mathrm{~K}$ SPH particles and each gas particle is first placed in the $x-y$ plane and given its circular velocity (determined by the dark matter halo) at its radius. The gas disk is then inclined by $\theta$ degrees with respect to the $x-y$ plane. An isothermal equation of state is used for the gas with a temperature of $1.2 \times 10^{3} \mathrm{~K}$ corresponding to a sound speed of $4 \mathrm{~km} \mathrm{~s}^{-1}$ ( $\sim 0.075$ times the virial velocity of the system). This extended and inclined gas disk is assumed to be dynamically affected only by a massive dark matter halo with the mass $M_{\mathrm{DM}}$ of $10^{10} M_{\odot}$ and we adopt the density distribution of the NFW halo (Navarro, Frenk \& White 1996) suggested from CDM simulations:

$$
\rho(r)=\frac{\rho_{0}}{\left(r / r_{\mathrm{s}}\right)\left(1+r / r_{\mathrm{s}}\right)^{2}},
$$

where $\rho_{0}$ and $r_{\mathrm{s}}$ are the central density and the scale length of a dark halo, respectively. We take the isodensity surfaces of the dark halo to be triaxial ellipsoids on which the Cartesian coordinates $(x, y, z)$ satisfy the following condition (Binney \& Tremaine 1987):

$$
m^{2} \equiv \frac{x^{2}}{a^{2}}+\frac{y^{2}}{b^{2}}+\frac{z^{2}}{c^{2}}=\text { constant }
$$

where $a, b$, and $c$ are the parameters which determine the two axis ratios of a triaxial body (i.e., long to short and long to middle). In the present study, $a$ is set to be 1 and the long-axis is initially coincident with the $x$ axis. Accordingly $b(\leq 1)$ and $c(\leq 1)$ are free parameters which determine the shapes of triaxial dark matter halos. The triaxial halo is assumed to be rotating as a solid body with the pattern speed of $\Omega_{\mathrm{p}}$. By changing the parameters $R_{\mathrm{g}}, \theta, b, c$, and $\Omega_{\mathrm{p}}$, we investigate morphological evolution of extended gas disks and its dependence on shapes and rotational properties of triaxial halos. The parameter values of $R_{\mathrm{g}}, \theta, b, c$, and $\Omega_{\mathrm{p}}$ are $15 \mathrm{kpc}, 30^{\circ}, 0.8,0.6$, and $3.84 \mathrm{~km} \mathrm{~s}^{-1} \mathrm{kpc}^{-1}$, respectively, in the standard model (Model 1). In the following, our units of mass, length, and time are $10^{10} \mathrm{M}_{\odot}\left(=\mathrm{M}_{\mathrm{DM}}\right), 15 \mathrm{kpc}$, and $2.74 \times 10^{8} \mathrm{yr}$, respectively.

Figure 1 describes how gaseous spiral arms are formed, as the triaxial halo rigidly rotates in the standard model (Model 1). Owing to the difference in angular speed between the gas disk and the triaxial halo, the gas disk continuously suffers from the strong tidal force of the halo. As a natural result of this, 


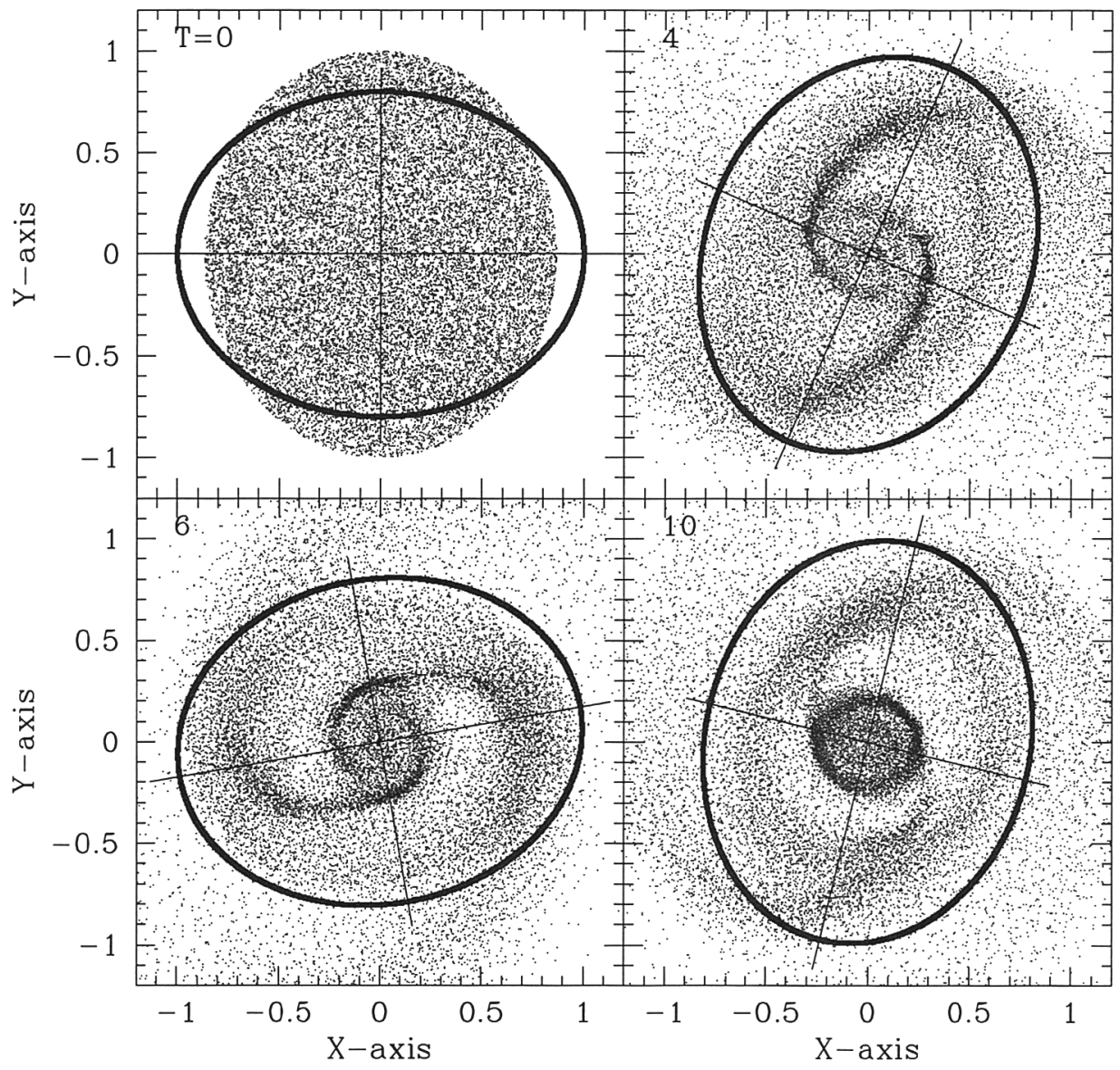

Figure 1. Morphological evolution of the gas disk projected onto the $x-y$ plane for the standard model with the disk inclination $(\theta)$ of $30^{\circ}$ with respect to the $x-y$ plane. The time $(T)$ indicated in the upper left corner of each frame is given in our units $\left(2.74 \times 10^{8} \mathrm{yr}\right)$ and each frame (2.4 in our units) measures $36 \mathrm{kpc}$ on a side. The shape of the triaxial halo projected onto the $x-y$ plane (at $R=1.0$ in our units, corresponding to $15 \mathrm{kpc}$ ) is outlined by a thick solid line at each time $T$. Both the gas disk and the halo are assumed to rotate counterclockwise (the spin axis of the halo is coincident with the $z$ axis). The long and middle axes of the halo are represented by thin solid lines. Note that as the triaxial halo rigidly rotates, two open trailing spiral arms are formed at $T=4.0(\sim 1.1 \mathrm{Gyr})$ owing to the tidal torque of the halo. Note also that these spirals arms finally wind to form a central gaseous ring at $T=10(\sim 2.7 \mathrm{Gyr})$. The points of ILR $\left(R_{\mathrm{ILR}}\right)$, corotation $\left(R_{\mathrm{CR}}\right)$, and outer Lindblad resonance $\left(R_{\mathrm{OLR}}\right)$ are $6.8,14.2$, and $20.7 \mathrm{kpc}$ for this standard model with $\Omega_{\mathrm{P}}=3.84 \mathrm{~km} \mathrm{~s}^{-1} \mathrm{kpc}^{-1}$. Triaxial dark matter halos with figure rotation can be formed after major merging between two NFW halos, if the remnant halos have larger spin parameters $(\lambda)$ of $>0.08$. 
two open trailing arms are gradually developed in the entire disk within $1 \mathrm{Gyr}$ $(T=4)$. These two open arms quickly wind with each other to form a central high-density, ring-like structure at $R \sim 0.3(4.5 \mathrm{kpc})$ in our units $(T=6$ and $8)$. Annular low-density gaseous regions $(0.3 \leq R \leq 0.5$ in our units) form just outside the inner ring because of the disk's angular momentum redistribution caused by the halo's torque $(T=10)$. Thus our simulations first confirm the early suggestion (Bureau et al. 1999) that the observed unusually extended spiral arms of NGC 2915 can be due to the tidal torque of the triaxial dark matter halo with figure rotation.

\section{Bar formation by triaxial dark halos with figure rotation}

Figure rotation of triaxial dark matter halos can significantly influence not only the formation of stellar bars, spiral arms, and warps but also gas dynamics and star formation histories in gas-rich galaxies (Bekki \& Freeman 2002; Bekki \& Freeman 2003, in preparation). Here we summarize briefly numerical results on stellar bar formation (hereafter "halo-triggered bar" formation) in disk galaxies embedded in triaxial (and spherically symmetric) dark matter halos with and without figure rotation (The details of the numerical models will be given in Bekki \& Freeman 2003). Based on fully self-consistent models (i.e., "live" halo, and self-gravitating exponential disks), we numerically investigated the shapes and the pattern speed of bars formed in disks embedded in dark matter halos with different pattern speeds of figure rotation, different radial density profiles (e.g., with or without central cusps/cores), different mass ratios of halos to disks $\left(f_{\mathrm{dm}}\right)$, and different shapes of the halos. See Fig. 2 for details of the models.

The results are summarized as follows: (1) Stellar bars can be formed in disks embedded in triaxial dark halos with figure rotation, even if $f_{\mathrm{dm}}$ is 5 , for which no bars can form in models with spherically symmetric halos, (2) Triaxial shapes and figure rotation disappear a few Gyr after the formation of stellar bars in the halos owing to the mutual dynamical interaction (i.e., dynamical friction) between disk and halo components, (3) Stellar bars are less likely to form in dark halos with cuspy radial distributions of the halos (i.e., bar formation is more likely in halos with the density profiles decribed by Salucci \& Burkert 2000), (4) The pattern speeds of the developed bars in triaxial dark matter halos with figure rotation and with larger $f_{\mathrm{dm}}(>10)$ are much lower than those of bars formed in a spontaneous way (i.e., due to bar instability) for massive disk models $\left(f_{\mathrm{dm}} \sim 2\right)$, and (5) Morphological properties of halo-triggered bars appear to be more similar to those of "tidal bars" (Noguchi \& Ishibashi 1986) that can be formed in interacting/merging galaxies.

\section{Implications: Halo-triggered starbursts and bar formation at high redshifts}

The above result (1) implies that bar formation is still possible in low luminosity LSBs that are observed to be dominated by dark matter halos, if the halos have figure rotation. If disk galaxies grow slowly by accretion of gas and satellite galaxies over $\sim 10 \mathrm{Gyr}$, (younger) high-z disk galaxies are likely to have larger $f_{\mathrm{dm}}$ and thus be less likely to develop stellar bars within the disks owing to spon- 


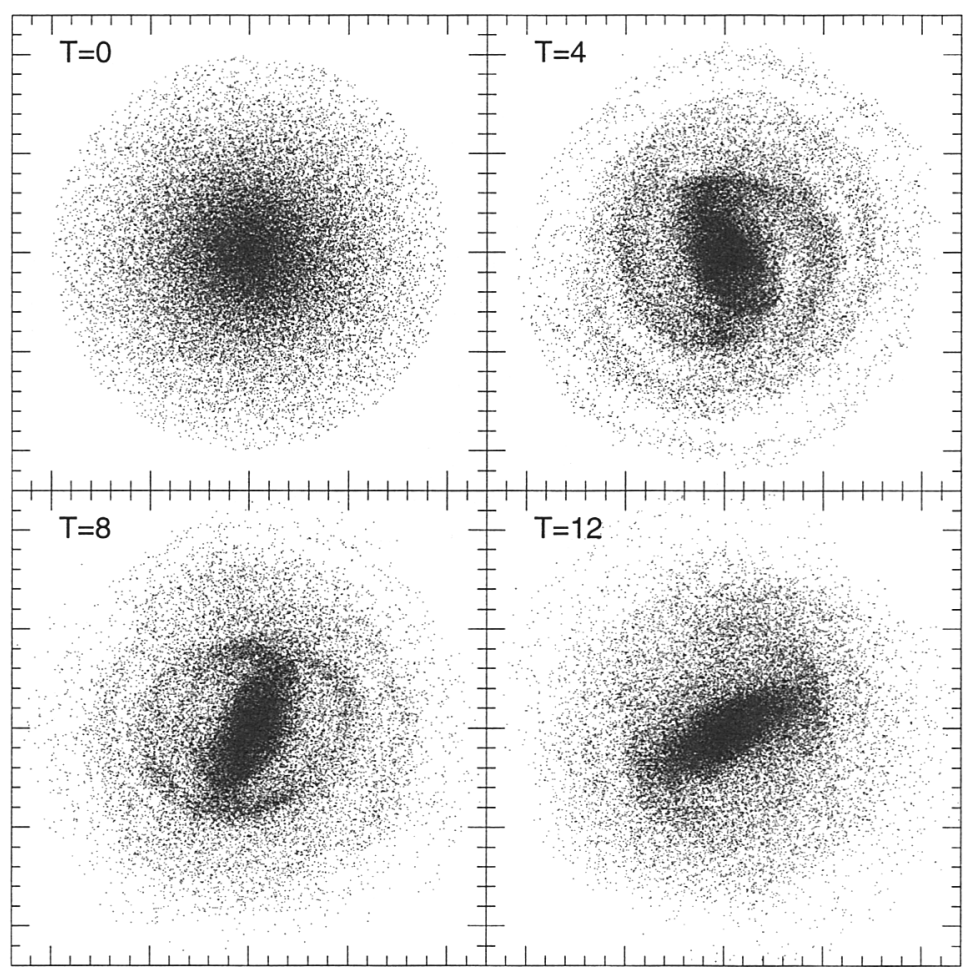

Figure 2. Morphological evolution of a stellar disk projected onto the $x-y$ plane for the disk galaxy model embedded in a massive dark matter halo with a triaxial shape and figure rotation. Triaxial dark halos with figure rotation can be constructed in the present numerical model based on numerical results on major merging between two axisymmetric spherical NFW (Navarro, Frenk \& White 1996) or SB (Salucci \& Burkert 2000) dark matter halos without figure rotation. In this model, the spin parameter $(\lambda)$ of the triaxial halo is $\sim 0.08$, which suggests that even if the spin parameter of a dark matter halo is not so large, the halo can have figure rotation. The time $(T)$ indicated in the upper left corner of each frame is given in our units $\left(1.41 \times 10^{8}\right.$ yr) and each frame (2.4 in our units) measures $42 \mathrm{kpc}$ on a side. Both the stellar disk and the halo are assumed to rotate counter-clockwise initially (the spin axis of the halo is coincident with the $z$ axis). The mass ratio of the triaxial dark halo to the disk $\left(f_{\mathrm{dm}}\right)$ is 5 within 2 disk radii and no bar can form in any spherically symmetric halo models with $f_{\mathrm{dm}}=5$. Therefore, the stellar bar seen in this figure is due to the tidal force of the massive triaxial halo with figure rotation for this model. The time scales of these halo-triggered bar formation in disks depend on $f_{\mathrm{dm}}$ and pattern speeds of figure rotation in the halos. 
taneous bar instability. Therefore, if high-z disk galaxies have bars, some of these disks could be embedded in triaxial dark halos with figure rotation. The above result (2) implies that lower-z dark halos are less likely to show triaxial shapes and figure rotation because of the long-term dynamical interaction between the halo-triggered bars and the halos. Regarding this point, BCD NGC 2915 and its triaxial halo could be formed relatively recently (though we can not specify the possible physical reasons for its late formation).

Non-axisymmetric barred structures (either in dark matter halos or in stellar disks) have been suggested to cause rapid and efficient radial gas transfer to the central regions of galaxies and thus be responsible for the formation of central starbursts (e.g., Noguchi \& Ishibashi 1986; Bekki \& Freeman 2002). Our numerical results accordingly suggest that (a) starbursts in galaxies can be triggered by tidal force of their triaxial dark halos with figure rotation and (b) such halo-triggered starbursts are possible even in the higher redshift universe where disk galaxies are less strongly self-gravitating and spontaneous bar formation is thus highly unlikely, if the halos have figure rotation. We therefore suggest that star formation histories of disk galaxies can be significantly different between those with and without triaxial shapes and figure rotation of dark matter halos at high redshifts. Lastly we stress that we have so far shown just two examples of the possible important roles of figure rotation of triaxial dark halos in galaxy evolution: Several important gravitational effects of these halos are yet to be explored such as dynamical effects of these halos on warp formation and on growth of central bulges in disk galaxies.

\section{References}

Bekki, K. 1997, ApJ, 483, 608

Bekki, K., \& Freeman, K. C. 2002, ApJ, 574, L21

Binney, J., \& Tremaine, S. 1987, Galactic Dynamics (Princeton: Princeton University Press)

Bureau, M., Freeman, K. C., Pfitzner, D. W., \& Meurer, G. R. 1999, AJ, 118, 2158

Franx, M., van Gorkom, J. H., \& de Zeeuw, T. 1994, ApJ, 436, 642

Navarro, J. F., Frenk, C. S., \& White, S. D. M. 1996, ApJ, 462, 563

Noguchi, M., \& Ishibashi, S. 1986, MNRAS, 219, 305

Olling, R. P. 1995, AJ, 110, 591

Sackett, P. D., \& Sparke, L. S. 1990, ApJ, 361, 408

Salucci, P., \& Persic, M. 1997, in Dark and Visible Matter in Galaxies, ed. M. Persic and P. Salucci ASP Conf. Ser. Vol. 117, p.1

Salucci, P., \& Burkert, A. 2000, ApJ, 537, L9 